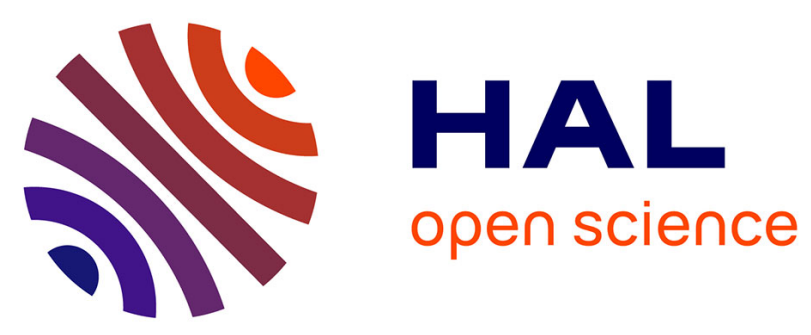

\title{
L'impact des mesures agri-environnementales sur le revenu des exploitations agricoles françaises
}

\author{
P. Berthelot, Vincent Chatellier, F. Colson
}

\section{To cite this version:}

P. Berthelot, Vincent Chatellier, F. Colson. L'impact des mesures agri-environnementales sur le revenu des exploitations agricoles françaises. Colloque: Les mesures agri-environnementales. Premiers bilans des expériences européennes, Société Française d'Economie Rurale (SFER). FRA., Nov 1997, Paris, France. 15 p. hal-02836958

\section{HAL Id: hal-02836958 \\ https://hal.inrae.fr/hal-02836958}

Submitted on 7 Jun 2020

HAL is a multi-disciplinary open access archive for the deposit and dissemination of scientific research documents, whether they are published or not. The documents may come from teaching and research institutions in France or abroad, or from public or private research centers.
L'archive ouverte pluridisciplinaire HAL, est destinée au dépôt et à la diffusion de documents scientifiques de niveau recherche, publiés ou non, émanant des établissements d'enseignement et de recherche français ou étrangers, des laboratoires publics ou privés. 


\section{Colloque SFER «Les mesures agri-environnementales »}

\section{- 3 et 4 Novembre 1997 -}

\section{L'impact des mesures agri-environnementales sur le revenu des exploitations agricoles françaises}

$--\cdot-\cdot-$

Philippe BERTHELOT - Vincent CHATELLIER - François COLSON (INRA ESR - Nantes)

\section{Introduction}

Pour encourager les exploitants agricoles à mettre en oeuvre ou à maintenir des pratiques de production plus compatibles avec la protection de l'environnement et la valorisation de l'espace rural, la réforme de la Politique agricole commune (PAC), adoptée en mai 1992, a été accompagnée d'un ensemble de mesures dites «agri-environnementales » (règlement communautaire européen $\mathrm{n}^{\circ} 2078$ du 30 juin 1992).

Les aides agri-environnementales, qui visent à prendre en compte les externalités positives de l'agriculture, représentent une faible part des aides directes versées au secteur agricole français (3 $\%$ en 1995). L'essentiel du soutien concerne les aides compensatoires des baisses de prix des céréales, des oléo-protéagineux et de la viande bovine, qui ne sont pas, pour l'instant, conditionnés $^{1}$ au respect de contraintes environnementales. Non plafonnés dans le secteur des grandes cultures et incitant à l'extensification dans les élevages de bovins viande, les paiements compensatoires de la réforme de la PAC sont à l'origine d'une accélération des rythmes d'agrandissement des exploitations agricoles. Dans ce contexte de concurrence entre les différents types de primes, les aides attribuées aux mesures agri-environnementales (MAE) ont eu peu d'influence sur les changements de pratiques (Gibault, 1996). Ces dernières, et tout particulièrement la prime à l'herbe, ont cependant joué un rôle important de complément de revenu dans les systèmes herbagers, notamment en zone de montagne.

Cette contribution cherche à vérifier, en fonction des différentes régions et des principales orientations de production, ce rôle de complément de revenu joué par les aides agrienvironnementales. Au préalable, la première partie rappelle les principales mesures agrienvironnementales adoptées en France et présente une rapide discussion sur l'intérêt et les limites

\footnotetext{
${ }^{1}$ Les contraintes de chargement imposées dans le secteur de l'élevage bovin - pour l'octroi des primes au maintien du troupeau de vaches allaitantes et des primes spéciales aux bovins mâles - ont été conçues comme des outils de maîtrise et de répartition des volumes de production et non comme des outils de limitation des risques de pollution.
} 
de la méthode d'analyse mise en oeuvre à partir des données du Réseau d'Information Comptable Agricole (RICA).

\section{1- Les mesures agri-environnementales et leur mode d'analyse à partir du RICA}

L'application des mesures agri-environnementales, obligatoire dans tous les États membres de l'Union européenne, fait l'objet de programmes agréés par la Commission. Au regard des prévisions budgétaires, les aides agri-environnementales sont concentrées principalement en Allemagne (1 005 millions d'Écus soit $32 \%$ du budget prévisionnel communautaire sur cinq ans), en France (660 millions d'Écus), en Italie (440 millions d'Écus) et en Espagne (394 millions d'Écus). Les trois nouveaux États membres (Autriche, Finlande, Suède) bénéficient pleinement de ces mesures avec un montant global de 841 millions d'Écus versés sur la seule année 1996.

Les aides agri-environnementales ne représentent qu'une très faible part des dépenses agricoles européennes (moins de 1\%), la majeure partie d'entre elles relevant du soutien direct et indirect des organisations communes de marché (céréales, lait, viande bovine). Le budget alloué aux mesures agri-environnementales repose sur le principe du cofinancement, l'Union européenne finançant, par l'intermédiaire de la section garantie du Fonds Européen d'Orientation et de Garantie Agricole (FEOGA), la moitié des dépenses (budget prévisionnel de 3158 millions d'écus sur la période 1993-1997) ${ }^{2}$. Sur la période 1993-1996, les dépenses effectives n'ont représenté que les deux tiers des sommes budgétisées, ce qui traduit la difficulté de mise en oeuvre de ces mesures (CNASEA, 1996).

\section{$\underline{\text { Une mesure nationale (prime à l'herbe) et des programmes régionaux }}$}

En France, les mesures agri-environnementales concernent deux niveaux géographiques d'intervention : un niveau national avec la prime au maintien des systèmes d'élevage extensifs (appelée «prime à l'herbe ») et les plans de développement durable ${ }^{3}$; un niveau régional avec les programmes zonaux et les opérations locales (tableau 1).

En France, la prime à l'herbe est sur le plan budgétaire la mesure agri-environnementale la plus importante. Destinée à encourager la désintensification de la production animale, le maintien des surfaces en herbe et l'entretien de prairies par les agriculteurs, cette mesure fait l'objet d'un

\footnotetext{
${ }^{2}$ Pour les régions en retard de développement, relevant de l'objectif 1 dans le cadre des fonds structurels communautaires (APCA, 1995), le financement du FEOGA est porté à $75 \%$.

${ }^{3}$ Les plans de développement durable sont assimilés à une mesure agri-environnementale gérée au niveau national. En phase d'expérimentation depuis 1995, ils concernent environ 800 agriculteurs (CNASEA, 1996). En encourageant la mise en place de nouveaux itinéraires techniques au sein des exploitations agricoles, ils cherchent à promouvoir la pérennité des entreprises dans un contexte de développement plus respectueux de l'environnement. Cette phase d'expérimentation fait suite à une étude de faisabilité portant, entre 1993 et 1994, sur 1250 exploitations réparties dans 59 sites.
} 
contrat entre l'agriculteur et la collectivité publique. Le bénéficiaire doit être exploitant à titre principal, né après le 31 décembre 1932, non retraité et non bénéficiaire d'une allocation de préretraite à la date du dépôt et pendant les cinq années d'engagement. Il doit en outre pouvoir justifier d'une exploitation d'au moins trois hectares et détenir en permanence au moins trois unités de gros bétail (UGB). Pour prétendre à cette prime, le taux de chargement de l'exploitation doit être inférieur à 1 UGB par hectare de superficie fourragère principale (SFP) ou 1,4 UGB par hectare dans le cas où la surface de prairies couvre plus $75 \%$ de la surface agricole. En 1995, le montant unitaire de la prime s'élève à 300 francs par hectare avec un plafond fixé à 100 hectares par exploitant ${ }^{4}$.

Tableau 1 : Les différents types de mesures agri-environnementales en France

\begin{tabular}{|c|c|c|}
\hline Niveau d'intervention & & Types de mesures \\
\hline \multirow[t]{2}{*}{$\underline{\text { Mesures nationales }}$} & \multicolumn{2}{|c|}{ Primes au maintien des systèmes d'élevages extensifs (ou prime à l'herbe) } \\
\hline & \multicolumn{2}{|c|}{ Plans de développement durable (expérimentation en cours) } \\
\hline \multirow[t]{3}{*}{$\underline{\text { Programmes régionaux }}$} & \multicolumn{2}{|c|}{ Extensification bovine et ovine par agrandissement } \\
\hline & \multicolumn{2}{|c|}{ Conversion à l'agriculture biologique } \\
\hline & Protection des & $\begin{array}{l}\text { Soutien aux races menacées de disparition } \\
\text { Reconversion des terres arables en herbages } \\
\text { extensifs }\end{array}$ \\
\hline \multirow[t]{5}{*}{ (cahiers des charges types) } & & Diminution des intrants \\
\hline & eaux & Lutte contre l'érosion \\
\hline & & Retrait à long terme \\
\hline & \multirow{2}{*}{$\begin{array}{l}\text { Protection de la } \\
\text { faune et de la flore }\end{array}$} & Jachère faunistique (ou retrait à long terme faune) \\
\hline & & Jachère floristique (ou retrait à long terme flore) \\
\hline (cahiers des charges spécifiques) & \multicolumn{2}{|c|}{ Opérations locales (anciennes OGAF-Environnement comprises) } \\
\hline
\end{tabular}

Sources : CNASEA - INRA Nantes

En juin 1996, la Commission européenne a approuvé, sur proposition française, vingt-quatre programmes régionaux, composés de deux grands types de mesures : les programmes zonaux qui font l'objet de cahiers des charges types et les opérations locales disposant de cahiers des charges spécifiques pour chaque opération (Pellegrini, 1996).

Les programmes zonaux comprennent cinq catégories de mesures : 1) L'extensification bovine ou ovine par agrandissement a pour objectif d'encourager les méthodes plus extensives d'élevage et de contribuer ainsi à lutter contre la déprise agricole. 2) Les aides à l'agriculture biologique permettent d'accompagner financièrement les agriculteurs dans le passage d'un système de production conventionnel à des méthodes de production conformes aux cahiers des charges de

\footnotetext{
${ }^{4}$ Les groupements pastoraux et associations foncières pastorales ne font pas l'objet de ce plafonnement. Dans les Groupements Agricoles d'Exploitation en Commun (GAEC), le plafond est multiplié par le nombre de parts.
} 
l'agriculture biologique. 3) Le soutien aux races menacées de disparition est destiné à maintenir et à protéger la biodiversité génétique des espèces animales (bovines, ovines, caprines, équines et asines). 4) Les aides à la protection des eaux et à la lutte contre les pollutions diffuses d'origine agricole visent à la reconversion des terres arables en herbages extensifs, à la diminution des intrants, à la lutte contre l'érosion et au retrait à long terme.

5) Les incitations à la protection de la faune et de la flore par la mise en place de jachère faunistique ou floristique.

Les opérations locales poursuivent un double objectif : l'adaptation des pratiques agricoles aux biotopes sensibles et rares ainsi que la gestion de zones de production très extensifiées, fragilisées par la déprise agricole ou menacées par des risques naturels. Elles s'inscrivent dans la continuité des opérations groupées d'aménagement foncier (OGAF - environnement), instaurées de 1989 à 1993 dans le cadre de l'article 19.

Les programmes régionaux français ont fait l'objet d'un budget prévisionnel global sur la période 1993-1997 de 2,8 milliards de francs (soit 558 millions de francs pendant cinq ans), financé pour moitié par l'Union Européenne ${ }^{5}$. Sept régions administratives concentrent plus de la moitié de cette enveloppe budgétaire : Pays de la Loire, Midi-Pyrénées, Centre, Rhône-Alpes, Basse-Normandie, Poitou-Charentes et Provence-Alpes-Côte d'Azur. La répartition des crédits entre les différentes mesures laisse apparaître le poids important des opérations locales (58\% du budget prévisionnel soit 328 millions de francs par an). Elles devancent budgétairement les mesures pour la protection des eaux $(21 \%)$, la conversion à l'agriculture biologique $(9,5 \%)$ et l'extensification par agrandissement $(9,5 \%)$. Les montants accordés aux autres mesures régionalisées restent très faibles.

Le taux d'engagement des programmes régionaux, qui correspond au rapport entre le montant des contrats signés et le budget prévisionnel, est relativement faible au terme de la quatrième année d'application (55\% contre $80 \%$ en théorie). Ce constat, qui se vérifie dans la plupart des régions à l'exception des Pays de la Loire, s'explique selon les enquêtes de terrain réalisées par une forte complexité des procédures et par une incitation financière peu attractive.

Le poids des aides agri-environnementales dans les aides directes à l'agriculture française

En 1995, les concours publics à l'agriculture productive s'élèvent en France à 71 milliards de francs, dont 50 milliards d'aides directes.

Tableau 2 : La part des aides agri-environnementales dans les aides directes versées à l'agriculture française (1995)

\footnotetext{
${ }^{5}$ L'inscription progressive des contractants au cours de la période 1993-1997 conduit à un étalement des versements au delà de l'année 1997.
} 


\begin{tabular}{||l||c|c||}
\hline & $\begin{array}{c}\text { Montants } \\
\text { (en millions de francs) }\end{array}$ & $\begin{array}{c}\text { Part en \% des aides } \\
\text { directes totales }\end{array}$ \\
\hline \hline Aides compensatoires aux cultures & 32662 & $65,3 \%$ \\
Aides bovines & 6604 & $13,2 \%$ \\
Aides agri-environnementales & 1520 & $3,0 \%$ \\
- dont primes à l'herbe & 1406 & $2,8 \%$ \\
- dont programmes régionaux & 114 & $0,2 \%$ \\
Aides montagne et zones défavorisées & 2496 & $5,0 \%$ \\
Autres aides & 6771 & $13,5 \%$ \\
\hline \hline Ensemble des aides directes & 50053 & $100,0 \%$ \\
\hline \hline
\end{tabular}

Sources : MAPA / CNASEA / INRA Nantes

Les aides compensatoires aux cultures (32,6 milliards de francs) et les primes bovines (6,6 milliards de francs) constituent la majeure partie de ces transferts (tableau 2). Les aides agrienvironnementales (1,5 milliards de francs dont plus de 1,4 milliards relèvent de la prime à l'herbe) représentent, quant à elles, seulement $3 \%$ de l'ensemble des aides directes.

Les aides agri-environnementales, comme les indemnités compensatoires de handicaps naturels (2,4 milliards de francs), sont une rémunération des agriculteurs en fonction de contraintes spécifiques (faible chargement, handicaps naturels). Elles peuvent aussi s'interpréter l'une et l'autre comme une rémunération des avantages environnementaux produits par l'agriculture. En étant ciblées vers les élevages extensifs, notamment ceux des zones difficiles, ces aides jouent un rôle important dans la formation du revenu des agriculteurs concernés, c'est ce que nous allons maintenant analyser de façon spécifique pour les aides agrienvironnementales.

\section{$\underline{\text { La méthode d'analyse des mesures agri-environnementales à partir du RICA }}$}

Pour caractériser les exploitations bénéficiant des aides agri-environnementales et mesurer la contribution des aides agri-environnementales à la formation du revenu des agriculteurs concernés par le dispositif, cet article s'appuie sur les données du Réseau d'Information Comptable Agricole (RICA). L'échantillon de 1995 (7232 exploitations) couvre, après application des coefficients individuels d'extrapolation, 428700 exploitations dites professionnelles ${ }^{6}$. Celles-ci représentent $58 \%$ de l'ensemble des exploitations agricoles françaises, détiennent $90 \%$ de la superficie agricole et assurent $95 \%$ de la production agricole finale. Le plan de sélection des exploitations selon la méthode des quotas (par région, orientation de production et classe de dimension économique) et la diversité des variables disponibles

${ }^{6}$. En France, une exploitation est considérée comme "professionnelle" dès lors qu'elle assure l'emploi d'au moins 0,75 Unité de Travail Annuelle (UTA) et que sa Marge Brute Standard (MBS) est supérieure à 8 Unités de Dimension Économique (soit un chiffre d'affaires voisin de 140000 francs). 
(techniques, économiques et financières) font de l'outil RICA un support pertinent pour réaliser ce travail.

En 1995, la création d'une variable permettant d'identifier le montant des aides directes agrienvironnementales autorise à un repérage précis des exploitations concernées par ces mesures. Les aides agri-environnementales étant globalisées au sein d'un même poste, aucune distinction n'est possible entre les différents types de mesures. La prime à l'herbe constituant près de $90 \%$ des crédits octroyés en 1995 et étant attribuée à la plupart des agriculteurs concernés par les autres mesures agri-environnementales, l'analyse porte en fait essentiellement sur les bénéficiaires de cette aide.

Au regard des données du RICA, 75300 exploitations agricoles bénéficient des MAE, soit nettement moins que le nombre de dossiers enregistrés par le CNASEA (prime à l'herbe). Le décalage entre les deux sources d'informations s'explique essentiellement par le fait que le champ du RICA est limité aux seules «exploitations professionnelles », alors que le CNASEA intègre dans ses calculs les 305000 exploitations agricoles considérées comme «non professionnelles » d'après les critères définis par le SCEES. Les estimations issues du RICA sont inférieures au CNASEA de $39 \%$ pour le nombre de bénéficiaires, de $18 \%$ pour le budget des aides agri-environnementales et de $15 \%$ pour les superficies éligibles. Le test par régions administratives (pour celles dont le nombre de bénéficiaires est suffisant pour permettre la comparaison) montre l'homogénéité du biais qui - moyennant le respect des règles habituelles de prudence sur l'interprétation des résultats - n'est pas un obstacle à l'utilisation de la base RICA pour une caractérisation des exploitations bénéficiaires des mesures agri-environnementales.

\section{2- Une exploitation agricole sur six a passé un contrat MAE}

En 1995, $17 \%$ des exploitations agricoles professionnelles françaises, soit 75300 unités, reçoivent des aides agri-environnementales. La répartition de ces exploitations est très inégale entre les régions et les principales orientations de production.

\section{Une concentration géographique en montagne}

Dans les zones de montagne et piémont, $60 \%$ des exploitations agricoles bénéficient du dispositif contre moins de $4 \%$ dans les zones de plaine. Cinq régions (Auvergne, Bourgogne, Limousin, Midi-Pyrénées et Rhône-Alpes) rassemblent $70 \%$ des exploitations contractantes et concentrent près des trois quarts du budget agri-environnemental national (tableau 3).

Tableau 3 : Les aides agri-environnementales selon les régions

\begin{tabular}{||c||c|c|c|c|c||}
\hline \hline & $\begin{array}{c}\text { Répartition du } \\
\text { budget MAE } \\
\text { (en \%) }\end{array}$ & $\begin{array}{c}\text { Aides MAE } \\
\text { / aides } \\
\text { directes } \\
\text { (en \%) }\end{array}$ & $\begin{array}{c}\% \text { des } \\
\text { exploitations } \\
\text { éligibles MAE }\end{array}$ & $\begin{array}{c}\text { Aides MAE (en F) } \\
\text { par exploitation } \\
\text { éligible aux MAE }\end{array}$ & $\begin{array}{c}\text { Aides MAE / } \\
\text { résultat courant par } \\
\text { exploitation éligible }\end{array}$ \\
\hline
\end{tabular}




\begin{tabular}{||l||c|c|c|c|c||} 
Auvergne & $26 \%$ & $14 \%$ & $71 \%$ & 21200 & $15 \%$ \\
Midi-Pyrénées & $14 \%$ & $4 \%$ & $32 \%$ & 13900 & $13 \%$ \\
Limousin & $13 \%$ & $11 \%$ & $70 \%$ & 18800 & $14 \%$ \\
Bourgogne & $11 \%$ & $4 \%$ & $35 \%$ & 22600 & $12 \%$ \\
Rhône-Alpes & $11 \%$ & $7 \%$ & $33 \%$ & 12100 & $10 \%$ \\
Aquitaine & $4 \%$ & $2 \%$ & $11 \%$ & 17500 & $14 \%$ \\
Franche-Comté & $4 \%$ & $7 \%$ & $36 \%$ & 18000 & $12 \%$ \\
L-Roussillon & $4 \%$ & $5 \%$ & $10 \%$ & 20000 & $15 \%$ \\
Pays de la Loire & $4 \%$ & $1 \%$ & $10 \%$ & 13800 & $7 \%$ \\
Autres régions & $9 \%$ & $\mathrm{~ns}$ & $\mathrm{~ns}$ & $\mathrm{~ns}$ & $\mathrm{~ns}$ \\
\hline \hline France & $100 \%$ & $3 \%$ & $17 \%$ & 16700 & $13 \%$ \\
\hline
\end{tabular}

Source : RICA France 1995 / INRA Nantes

En Auvergne et dans le Limousin, sept exploitations sur dix bénéficient des aides agri-environnementales, pour un montant moyen par exploitation éligible de respectivement 21200 francs (soit $15 \%$ du résultat courant) et 18800 francs (soit $14 \%$ du résultat courant). Ces aides, composées essentiellement de la prime à l'herbe, représentent $14 \%$ de l'ensemble des aides directes attribuées à l'Auvergne, $11 \%$ en Limousin, $7 \%$ en Rhône-Alpes et en FrancheComté et moins de $5 \%$ pour toutes les autres régions françaises.

Dans les exploitations bénéficiaires, les aides agri-environnementales représentent, en moyenne nationale, $13 \%$ du résultat courant, cette contribution étant peu différente selon les régions, à l'exception des Pays de la Loire où elles ne couvrent que $7 \%$ du revenu.

\section{Les exploitations spécialisées en viande bovine sont fortement concernées}

L'analyse par orientation de production souligne la forte concentration des aides agri-environnementales dans les exploitations spécialisées en production de viande bovine, qui représentent $10 \%$ de l'ensemble des exploitations agricoles mais rassemblent $41 \%$ du budget agri-environnemental, contre $30 \%$ pour les exploitations de l'orientation Elevage et cultures et $20 \%$ pour celles spécialisées en production laitière ${ }^{7}$ (tableau 4).

Tableau 4 : Les aides agri-environnementales selon les orientations de production

\footnotetext{
${ }^{7}$ La répartition des exploitations selon les types de production repose sur des regroupements d'orientations de production (OTEX) : Viande bovine (OTEX 42) ; Elevage et cultures (OTEX 44+60+71+81+82); Lait spécialisé (OTEX 41); Grandes cultures (OTEX 13+14).
} 


\begin{tabular}{||l||c|c|c|c|c||}
\hline \hline & $\begin{array}{c}\text { Répartition du } \\
\text { budget MAE } \\
(\mathrm{en} \%)\end{array}$ & $\begin{array}{c}\text { Aides MAE } \\
\text { / aides } \\
\text { directes } \\
\text { (en } \%)\end{array}$ & $\begin{array}{c}\% \text { des } \\
\text { exploitations } \\
\text { éligibles MAE }\end{array}$ & $\begin{array}{c}\text { Aides MAE (en F) } \\
\text { par exploitation } \\
\text { éligible aux MAE }\end{array}$ & $\begin{array}{c}\text { Aides MAE / } \\
\text { résultat courant par } \\
\text { exploitation éligible }\end{array}$ \\
\hline \hline Viande bovine & $41 \%$ & $10 \%$ & $55 \%$ & 22000 & $16 \%$ \\
Elevage et cultures & $30 \%$ & $3 \%$ & $24 \%$ & 15400 & $10 \%$ \\
Lait spécialisé & $20 \%$ & $6 \%$ & $24 \%$ & 13300 & $10 \%$ \\
Lait viande & $6 \%$ & $4 \%$ & $24 \%$ & 17000 & $12 \%$ \\
Grandes cultures & $2 \%$ & $0 \%$ & $2 \%$ & 10700 & $6 \%$ \\
Autres orientations & $1 \%$ & $\mathrm{~ns}$ & $2 \%$ & $16 \%$ & $\mathrm{~ns}$ \\
\hline \hline Ensemble & $100 \%$ & $3 \%$ & $17 \%$ & 100 & $13 \%$ \\
\hline
\end{tabular}

Source : RICA France 1995 / INRA Nantes

Les faibles niveaux d'intensification des superficies fourragères dans certains systèmes allaitants conduisent à une proportion importante d'exploitations éligibles aux aides agrienvironnementales au sein de l'orientation viande bovine (55\% contre $24 \%$ pour les trois autres groupes d'orientations relatifs à l'élevage). Les aides agri-environnementales représentent, en moyenne, $10 \%$ des aides directes attribuées aux 42500 exploitations spécialisées en production de viande bovine contre seulement $6 \%$ pour les 76200 exploitations laitières. Pour ces dernières, les aides aux superficies de maïs ensilage sont, en moyenne nationale, six fois plus élevées que la prime à l'herbe.

\section{Les exploitations éligibles aux MAE regroupent $42 \%$ de la surface toujours en herbe}

Les 75300 exploitations bénéficiaires des mesures agri-environnementales détiennent $20 \%$ de la superficie agricole nationale. Spécialisées dans l'élevage bovin, elles occupent $43 \%$ de la superficie toujours en herbe et $35 \%$ de la superficie fourragère principale. Elles sont peu concernées par la production de maïs fourrage (10\% des superficies) et par les cultures de céréales et d'oléo-protéagineux (6\%). La superficie agricole moyenne par exploitation bénéficiaire est plus grande que celle des non bénéficiaires (67 hectares contre 58 hectares), l'assolement est constitué pour près des quatre cinquièmes de surfaces fourragères, les deux tiers de celles-ci correspondant à des prairies permanentes (tableau 5).

Tableau 5 : Le poids des exploitations agricoles bénéficiaires des MAE

\begin{tabular}{||l||c|c||r|r||}
\hline \hline & \multirow{2}{*}{$\begin{array}{c}\text { Exploitations } \\
\text { sans MAE }\end{array}$} & $\begin{array}{c}\text { Exploitations } \\
\text { avec MAE }\end{array}$ & \multicolumn{2}{|c||}{ Ensemble des exploitations } \\
\cline { 3 - 5 } & (en \%) & $17 \%$ & (somme) \\
\hline \hline Nombre d'exploitations agricoles & $83 \%$ & $17 \%$ & 428700 \\
Unité de travail agricole & $85 \%$ & $15 \%$ & $100 \%$ & 767400 \\
Superficie agricole utile & $80 \%$ & $20 \%$ & $100 \%$ & 25520000 ha \\
Superficie COP (hors maïs fourrage) & $94 \%$ & $6 \% \%$ & 11725000 ha \\
Superficie fourragère & $65 \%$ & $35 \%$ & $100 \%$ & 11150000 ha \\
- dont surface toujours en herbe & $57 \%$ & $43 \%$ & $100 \%$ & 5990000 ha
\end{tabular}




\begin{tabular}{||l||l|r||r|r||} 
- dont maïs fourrage & $90 \%$ & $10 \%$ & $100 \%$ & 1680000 ha \\
\hline UGB herbivores & $71 \%$ & $29 \%$ & $100 \%$ & 15550000 \\
- dont vaches laitières & $83 \%$ & $17 \%$ & $100 \%$ & 4617000 \\
- dont vaches allaitantes & $58 \%$ & $42 \%$ & $100 \%$ & 3794000 \\
\hline Production agricole & $91 \%$ & $9 \%$ & $100 \%$ & 266590 Mio F \\
Valeur ajoutée brute & $92 \%$ & $8 \%$ & $100 \%$ & 103750 Mio F \\
Résultat courant & $88 \%$ & $12 \%$ & $100 \%$ & 80250 Mio F \\
\hline Aides directes totales & $82 \%$ & $18 \%$ & $100 \%$ & 46840 Mio F \\
- dont aides COP & $95 \%$ & $5 \%$ & $100 \%$ & 32230 Mio F \\
- dont aides handicaps naturels & $28 \%$ & $72 \%$ & $100 \%$ & 1800 Mio F \\
\hline \hline
\end{tabular}

Source : RICA France 1995 / INRA Nantes

Près de $42 \%$ du cheptel de vaches allaitantes et $17 \%$ du cheptel de vaches laitières sont concentrés dans des exploitations éligibles aux aides agri-environnementales. La concentration importante de la production laitière dans les régions de l'Ouest de la France (Bretagne, Pays de la Loire, Basse-Normandie) où le chargement bovin par hectare de superficie fourragère est élevé du fait notamment de la présence de maïs fourrage - conduit à ce que $83 \%$ des vaches laitières soient répertoriées dans des unités non éligibles aux aides agri-environnementales.

Les exploitations bénéficiaires des aides agri-environnementales ont une dimension économique plus faible que les autres unités (58\% d'entre elles ont un chiffre d'affaires inférieur à $300000 \mathrm{~F}$ contre $32 \%$ dans l'autre cas). En représentant $17 \%$ des exploitations agricoles, elles assurent seulement $9 \%$ de la production agricole nationale, $8 \%$ de la valeur ajoutée brute et $12 \%$ du résultat courant. En ayant une production agricole moyenne par exploitation deux fois plus réduite que les autres (330 300 francs contre 684000 francs), les exploitations éligibles au dispositif disposent d'un montant moyen d'aides directes comparable (environ 110000 francs par exploitation soit respectivement $85 \%$ et $55 \%$ du résultat courant).

Le montant moyen d'aides directes est comparable entre les deux groupes étudiés, mais les postes de subventions diffèrent fortement, en raison notamment de la localisation géographique et de la spécialisation agricole des exploitations. Dans les exploitations bénéficiaires des aides agrienvironnementales, les aides directes sont constituées pour $31 \%$ de primes bovines (primes au maintien du troupeau de vaches allaitantes et primes spéciales aux bovins mâles), pour $21 \%$ d'aides sur les superficies de céréales et d'oléo-protéagineux, pour $15 \%$ d'aides agrienvironnementales, pour $15 \%$ d'indemnités compensatoires de handicaps naturels, pour $11 \%$ de primes compensatrices ovines et pour $7 \%$ d'autres aides directes (accidents climatiques, aides locales et régionales, etc...). Dans les exploitations non éligibles, les aides COP représentent $79 \%$ de l'enveloppe contre $11 \%$ pour les primes bovines et seulement $10 \%$ pour l'ensemble des autres aides.

\section{3- Un impact limité sur le revenu des exploitations en contrat MAE}


L'impact des aides agri-environnementales sur le revenu est très différent selon les exploitations. Parmi les exploitations éligibles, les aides MAE représentent moins de $10 \%$ du résultat courant dans un tiers des unités et plus de $30 \%$ dans seulement $18 \%$ d'entre elles (tableau 6).

Tableau 6 : Les caractéristiques moyennes des exploitations éligibles aux MAE selon le poids des aides agri-environnementales dans le résultat courant

\begin{tabular}{||l||r|r|r|r||r||}
\hline & \multicolumn{1}{|c|}{$0-10 \%$} & $10-20 \%$ & $20-30 \%$ & $>30 \%$ & Ensemble \\
\hline \hline Nombre d'exploitations & 25000 & 28700 & 8400 & 13200 & 75300 \\
\hline Unité de travail agricole & 1,72 & 1,43 & 1,48 & 1,35 & 1,52 \\
UGB herbivores & 59 & 63 & 59 & 51 & 60 \\
Superficie agricole (ha) & 65 & 69 & 64 & 61 & 67 \\
Superficie COP (hors maïs) & 14 & 7 & 6 & 7 & 10 \\
Superficie fourragère & 47 & 57 & 47 & 51 & 52 \\
\hline Production agricole (F) & 463900 & 306200 & 239700 & 187000 & 330300 \\
Valeur ajoutée brute & 183300 & 97100 & 54300 & 3500 & 104600 \\
Résultat courant & 203900 & 128800 & 89700 & 22700 & 130800 \\
\hline Aides directes totales & 113200 & 110700 & 113500 & 104700 & 112100 \\
- dont aides MAE & 11000 & 17400 & 20900 & 22500 & 16700 \\
Aides directes / ha de SAU & 1750 & 1590 & 1760 & 1700 & 1680 \\
Aides MAE / ha de SFP & 230 & 305 & 440 & 450 & 320 \\
\hline
\end{tabular}

Source : RICA France 1995 / INRA Nantes

La dépendance à l'égard des aides agri-environnementales (en pourcentage du revenu et en montant d'aides par hectare) est d'autant plus forte que la production agricole de l'exploitation est limitée et que le résultat courant est faible. Les 13200 exploitations agricoles pour lesquelles les aides agri-environnementales représentent plus de $30 \%$ du résultat courant dégagent, en moyenne, une production agricole limitée (187 000 francs) et un résultat courant faible (22 700 francs). Dans ces exploitations, les aides agri-environnementales entrent pour $22 \%$ dans le montant total des aides directes, qui représente plus de la moitié de la production agricole (en valeur). Les 25000 exploitations pour lesquelles les aides agri-environnementales représentent moins de $10 \%$ du résultat courant ont une production agricole trois fois plus importante que ces dernières (463900 francs) mais un montant total d'aides directes voisin tant par exploitation (113 200 francs) que par hectare $(1750 \mathrm{~F})$.

Les aides agri-environnementales : un complément de revenu en production de viande bovine

Parmi les 42500 exploitations spécialisées en production de viande bovine (OTEX 42), $55 \%$, soit 23500 unités, bénéficient des aides agri-environnementales (tableau 7). Ces exploitations se 
différencient des autres unités par une superficie moyenne plus importante ( 77 hectares contre 57 hectares) et un niveau moyen de chargement herbivore nettement plus faible (1,13 UGB herbivores par hectare de surface fourragère contre 1,55). Dans les deux groupes, la superficie fourragère couvre près de $85 \%$ de la superficie agricole, les prairies permanentes étant plus représentées dans les exploitations bénéficiaires des mesures agri-environnementales $(70 \%$ de la surface fourragère contre $50 \%$ ).

Tableau 7 : Les caractéristiques moyennes des exploitations spécialisées en viande bovine

\begin{tabular}{|c|c|c|c|}
\hline & $\begin{array}{c}\text { Exploitations } \\
\text { sans MAE }\end{array}$ & $\begin{array}{c}\text { Exploitations } \\
\text { avec MAE }\end{array}$ & Ensemble \\
\hline Nombre d'exploitations agricoles & 19000 & 23500 & 42500 \\
\hline Unité de travail agricole & 1,36 & 1,41 & 1,38 \\
\hline UGB herbivores & 75 & 77 & 76 \\
\hline UGB herbivores / ha de SFP & 1,55 & 1,13 & 1,28 \\
\hline Superficie agricole utile (ha) & 57 & 77 & 68 \\
\hline Superficie COP (hors maïs fourrage) & 8 & 6 & 7 \\
\hline Superficie fourragère & 48 & 68 & 59 \\
\hline - dont surface toujours en herbe & 24 & 47 & 37 \\
\hline - dont surface de maïs fourrage & 5 & 1 & 3 \\
\hline Production agricole & 306000 & 261500 & 281500 \\
\hline Valeur ajoutée brute & 91900 & 70500 & 80100 \\
\hline Résultat courant & 125100 & 134300 & 130200 \\
\hline
\end{tabular}

Source : RICA France 1995 / INRA Nantes

Les exploitations spécialisées en viande bovine qui bénéficient des aides agrienvironnementales dégagent, compte tenu notamment d'un niveau plus élevé d'aides directes (139000 francs contre 109000 francs), un résultat courant supérieur aux autres unités de la même orientation (134 300 francs contre 125100 francs).

Le montant moyen des aides agri-environnementales par exploitation éligible s'élève à 21900 francs (tableau 8), soit un montant largement supérieur au différentiel d'aides observé sur les superficies de maïs fourrage (+ 6900 francs au profit des unités ne bénéficiant pas des aides agrienvironnementales).

Tableau 8 : Les aides directes dans les exploitations spécialisées en viande bovine

\begin{tabular}{|l|l|l||c|}
\hline \hline & Exploitations & Exploitations & Ensemble \\
\hline
\end{tabular}




\begin{tabular}{|c|c|c|c|}
\hline & sans MAE & avec MAE & \\
\hline Aides directes totales & 109000 & 139000 & 125600 \\
\hline Aides SCOP (hors maïs ensilage) & 12900 & 9500 & 11100 \\
\hline Aides à l'élevage & 82800 & 118400 & 102300 \\
\hline - dont primes animales (PMTVA+PSBM+PCO) & 76200 & 84600 & 80900 \\
\hline - dont aides maïs ensilage (estimation) & 9300 & 2400 & 5500 \\
\hline - dont aides agri-environnementales & 0 & 21900 & 12100 \\
\hline - dont aides pour handicaps géographiques & 5900 & 14900 & 10900 \\
\hline Aides directes par UTA & 80200 & 98600 & 91000 \\
\hline Aides directes / EBE (en \%) & $56 \%$ & $69 \%$ & $63 \%$ \\
\hline Aides directes par ha de SAU & 1890 & 1810 & 1840 \\
\hline Aides à l'élevage par ha de SFP & 1890 & 1820 & 1850 \\
\hline - dont aides MAE par ha de SFP & 0 & 320 & 200 \\
\hline - dont aides ICHN par ha de SFP & 120 & 220 & 180 \\
\hline
\end{tabular}

Source : RICA France 1995 / INRA Nantes

Du fait du versement des aides MAE et ICHN, le montant total des aides directes par hectare de SAU et le montant des aides à l'élevage par hectare de SFP est relativement proche entre les deux groupes étudiés. Notons également que les écarts de revenu qui existaient avant la réforme de la PAC entre les exploitations bovines intensives et extensives ont progressivement disparu du fait de l'instauration de la prime à l'herbe et du complément versé aux élevages extensifs $(+30$ Écus par UGB dans les exploitations ayant un facteur de densité inférieur à 1,4 UGB PAC par hectare de SFP).

\section{$\underline{\text { Un quart des exploitations laitières bénéficient des aides agri-environnementales }}$}

Sur les 76200 exploitations laitières spécialisées, $24 \%$ (soit 18800 unités) reçoivent des aides au titre des mesures agri-environnementales. Moins intensives (1,1 UGB herbivores par ha de SFP), les exploitations laitières éligibles au dispositif ont une superficie fourragère plus élevée (45 hectares) et une superficie agricole équivalente aux autres (tableau 9).

Tableau 9 : Les caractéristiques moyennes des exploitations laitières spécialisées

\begin{tabular}{||l||r|r||r||}
\hline & $\begin{array}{c}\text { Exploitations } \\
\text { sans MAE }\end{array}$ & $\begin{array}{c}\text { Exploitations } \\
\text { avec MAE }\end{array}$ & Ensemble \\
\hline \hline Nombre d'exploitations agricoles & 57400 & 18800 & 76200 \\
\hline Unité de travail agricole & 1,61 & 1,48 & 1,58 \\
Quota laitier (kg) & 198900 & 142700 & 185000 \\
UGB herbivores & 64 & 1,10 & 60 \\
UGB herbivores / ha de SFP & 1,56 & 1,43
\end{tabular}




\begin{tabular}{||l||r|r||r||} 
Superficie agricole utile (ha) & 51 & 52 & 51 \\
Superficie COP (hors maïs fourrage) & 9 & 4 & 8 \\
Superficie fourragère & 41 & 45 & 42 \\
- dont surface toujours en herbe & 18 & 32 & 22 \\
- dont surface de maïs fourrage & 11 & 3 & 9 \\
\hline Production agricole & 583900 & 374200 & 532200 \\
Valeur ajouté brute & 225800 & 151100 & 207400 \\
Résultat courant & 172800 & 124500 & 160900 \\
\hline
\end{tabular}

Source : RICA France 1995 / INRA Nantes

Localisées principalement en Auvergne, en Rhône-Alpes et en Franche-Comté, elles ont un cheptel moyen plus limité (49 UGB herbivores contre 64) et un quota laitier plus modeste (142700 kg contre $198900 \mathrm{~kg}$ ). Les superficies de prairies permanentes représentent $61 \%$ de leur assolement contre $35 \%$ pour les exploitations non concernées par la prime à l'herbe. Ces dernières cultivent, en revanche, 11 hectares de maïs fourrage (contre seulement 3 hectares dans le premier cas), soit un supplément d'aides maïs (16 200 francs) inférieur au différentiel d'aides MAE et ICHN (27 500 francs) existant entre les deux groupes étudiés (tableau 10).

Contrairement à ce qui est observé pour les unités spécialisées en viande bovine où les contraintes de chargement s'appliquent, les exploitations laitières éligibles aux aides MAE bénéficient d'un montant plus faible d'aides directes (52 700 francs contre 55200 francs). Rapportées à l'actif agricole ou à l'hectare de SAU, le montant des aides est cependant très proche entre les deux groupes ${ }^{8}$. Dans les exploitations bénéficiaires, le montant global d'aides provient pour $33 \%$ des indemnités compensatoires de handicaps naturels, pour $25 \%$ des aides agri-environnementales, pour $22 \%$ des aides COP, pour $11 \%$ de la prime spéciale aux bovins mâles et pour $9 \%$ des autres aides directes. Dans les autres exploitations, les aides COP représentent $72 \%$ du montant global des aides.

Tableau 10 : Les aides directes dans les exploitations laitières spécialisées

\begin{tabular}{||l||c|c||c||}
\hline \hline & $\begin{array}{c}\text { Exploitations } \\
\text { sans MAE }\end{array}$ & $\begin{array}{c}\text { Exploitations } \\
\text { avec MAE }\end{array}$ & Ensemble \\
\hline \hline Aides directes totales & 55200 & 52700 & 54600 \\
\hline
\end{tabular}

\footnotetext{
${ }^{8}$ La prise en compte d'un équivalent soutien direct lié au quota conduirait à un soutien par hectare et par actif plus important dans les unités non éligibles. Ces dernières produisent, en effet, $4850 \mathrm{~kg}$ de lait par hectare de SFP contre $3200 \mathrm{~kg}$ pour les autres.
} 


\begin{tabular}{||l||r|r||r||} 
Aides SCOP (hors maïs ensilage) & 17900 & 6700 & 15100 \\
Aides à l'élevage & 32800 & 42300 & 35100 \\
- dont primes animales (PMTVA+PSBM+PCO) & 7900 & 6200 & 7500 \\
- dont aides au maïs ensilage & 21800 & 5600 & 17800 \\
- dont aides agri-environnementales & 0 & 13100 & 3200 \\
- dont aides pour handicaps géographiques & 3100 & 17500 & 6600 \\
\hline Aides directes par UTA & 34300 & 35600 & 34600 \\
Aides directes / EBE (en \%) & $20 \%$ & $27 \%$ & $22 \%$ \\
Aides directes par ha de SAU & 1090 & 1010 & 1070 \\
Aides à l'élevage par ha de SFP & 800 & 930 & 840 \\
- dont aides MAE par ha de SFP & 0 & 290 & 80 \\
- dont aides ICHN par ha de SFP & 70 & 390 & 160 \\
\hline
\end{tabular}

Finalement, les écarts importants de dimension économique font que les exploitations laitières bénéficiant des aides agri-environnementales ont, en moyenne, un résultat courant nettement inférieur aux autres (124 500 francs contre 172800 francs).

\section{Conclusion}

Au regard des données nationales du RICA, $17 \%$ de l'ensemble des exploitations agricoles "professionnelles" ont bénéficié des aides agri-environnementales. Ces aides, qui relèvent pour plus de $90 \%$ de la prime à l'herbe, sont très fortement concentrées dans les exploitations des zones de montagne (60\% de l'enveloppe budgétaire). Plus de la moitié des exploitations de l'orientation viande bovine en bénéficient contre un quart pour celles des orientations bovins lait, élevage-cultures, lait viande et moins de $2 \%$ pour toutes les autres orientations de production.

La dimension économique des exploitations contractantes est assez limitée (58\% ont un chiffre d'affaires inférieur à 300000 francs), même si les superficies sont, en moyenne, plus importantes que dans les autres structures. Pour les exploitations éligibles, les aides agrienvironnementales représentent $15 \%$ du résultat courant, lequel reste largement plus faible que dans les autres exploitations (106 500 francs contre 156400 francs). En étant particulièrement ciblées, les aides agri-environnementales contribuent, avec les indemnités compensatoires de handicaps géographiques, à assurer un complément de revenu qui contribue à la pérennité de certaines exploitations bovines situées en zones difficiles.

En soutenant les exploitations les plus extensives, les mesures agri-environnementales, et en particulier la prime à l'herbe, contribuent de façon ciblée au maintien de pratiques agricoles respectueuses de l'environnement. Le poids financier de ces mesures demeure cependant très 
faible comparativement aux paiements compensatoires attribués (sans plafonnement ou conditionnalité environnementale) via les mécanismes de la Politique agricole commune.

Les propositions récentes d'adaptation de la PAC faites dans le cadre d'Agenda 2000 ne sont pas encore suffisamment précises pour que leur portée environnementale puisse être appréciée. La poursuite, en l'état, du dispositif agri-environnemental ne sera vraisemblablement pas suffisante, à elle seule, pour orienter l'agriculture française et européenne vers des pratiques agricoles plus extensives et pour rémunérer les aménités positives de l'agriculture. Une telle évolution impliquerait une conditionnalité sociale et environnementale de l'ensemble des aides compensatoires au revenu agricole. Ce sera l'un des enjeux des négociations à venir, non seulement dans le cadre de l'Union Européenne pour la réforme de la PAC et les futurs accords de l'organisation mondiale du commerce (OMC), mais aussi entre les représentants des organismes professionnels agricoles et ceux des autres catégories sociales. 


\section{Bibliographie}

Amand-Madelin V. La prise en compte de l'environnement dans les politiques agricoles. In Économie et statistique $\mathrm{n}^{\circ} 258-259$, 1992, pp 105-112.

Assemblée Permanente des Chambres d'Agriculture. Les fonds structurels communautaires. In Chambres d'Agriculture, n829, janvier 1995, 48 p.

Boisson J.M. et Buller H. The European experiences of policies for the Agricultural Environment. 40th EAAE Seminar, Ancona, Italie, 26-28 juin 1995, 34 p.

Caron P. La question de l'environnement : ferment d'innovations radicales pour la Politique agricole commune. In Courrier de l’Environnement n²5, 1995, pp 71-76.

Colson F., Chatellier V. Réforme de la PAC et répartition des paiements directs entre les exploitations agricoles françaises. In Économie Rurale $\mathrm{n}^{\circ} 227$, mars-juin 1995, pp 11-20.

CNASEA : Rapport d'activités 1996. CNASEA, Paris, pp 56 à 64.

Communautés européennes. Règlement $(\boldsymbol{C E E}) \boldsymbol{n}^{\circ}$ 2078/92. Journal officiel des CE, 30 juillet 1992, pp 85 à 89.

Dobremez L., Véron F., 1997 : Contribution à l'évaluation des mesures agrienvironnementales : exemples de démarches. In Ingénieries n¹0, juin 1997, pp 3-15.

Gibault I. L'application des mesures agri-environnementales dans les zones humides des Pays de la Loire. Mémoire DESS, septembre 1996, 85 p.

Ministère de l'Agriculture de la Pêche et de l'Alimentation. Les concours publics à l'agriculture 1991-1995. Paris, octobre 1996, 195 p.

Ministère de l'Agriculture de la Pêche et de l'Alimentation. Mesures agri-environnement Innovation et tradition. Paris, BIMA, $\mathrm{n}^{\circ} 1449$, mars 1996, pp 29-32.

Pellegrini N. Les mesures agri-environnementales. In Courrier de l'Environnement de l'INRA, $\mathrm{n}^{\circ} 25$, septembre 1996, pp 128-129.

\section{Sigles}

CNASEA : Centre National d'Aménagement des Structures d'Exploitations Agricoles

FEOGA : Fonds Européen d'Orientation et de Garantie Agricole

ICHN : Indemnités Compensatoires de Handicaps Naturels

MAE : Mesures Agri-Environnementales

OGAF : Opérations Groupées d'Aménagement Foncier 
RICA : Réseau d'Information Comptable Agricole

SCEES : Service Central des Études Économiques et Statistiques

SCOP : Superficie en Céréales et Oléo-Protéagineux

SFP : Superficie Fourragère Principale

UGB : Unité de Gros Bétail 